\title{
EFFECT OF ECHINACEA PURPUREA AND GARLIC ON GROWTH PERFORMANCE, IMMUNE RESPONSE, BIOCHEMICAL AND HEMATOLOGICAL PARAMETERS IN BROILER CHICKS
}

\author{
MAISA M. GHARIEB and FATMA M. YOUSSEF \\ Animal health Research Institute-Ismailia \\ Email: dr-mmg63@yahoo.com
}

\section{ABSTRACT}

Received at: 22/12/2013

This study was conducted to investigate the effect of Echinacea purpurea and garlic on growth performance, immune response, biochemical and hematological parameters in broiler chick. A total of (180) chickens were divided into 3 main groups A,B and C. First group as control group received only commercial basal diet, second group was fed on commercial basal diet

Accepted: 23/2/2014 plus Echinacea purpurea as $5 \mathrm{mg} / \mathrm{kg}$ feed and the third group was fed on commercial basal diet plus garlic powder as $1 \mathrm{~g} / \mathrm{kg}$ feed. All the other conditions were the same for all the groups. At $21^{\text {th }}$ days of age, each group was subdivided into two groups as group A: subgroups G1 and G2, group B: subgroups G3 and G4 and group C: subgroups G5 and G6. Groups G2,G4 and G6were challenged with $0.5 \mathrm{ml}$ E. coli $\mathrm{O} 78$ (4 $\left.\mathrm{x} 10^{6} \mathrm{CFU}\right)$ by intraperitoneal route. Performance, immunity system and some blood parameters were measured. As well as clinical signs, postmortem and mortality were recorded. Experimentally infected and treated chickens with Echinacea purpurea and garlic powder displayed less symptoms with the mortality rate of $16.66 \%(5 / 30)$ in comparison to infected non treated group with mortality rate of $40 \%$ (12/30). The postmortem lesions were reduced to greater extent as compared to infected untreated birds. There were significant differences in Echinacea purpurea and garlic treated groups with control in body weight gain, feed conversion ratio from performance, heterophils/ lymphocytes ratio, lymphocyte stimulation index, albumin and protein differentiation from immune system, and triglyceride from biochemical parameters. Cholesterol level was lower in garlic and Echinacea purpurea groups. According to the results, Echinacea purpurea and garlic can be used as a good alternative for commercial antibiotics, growthpromoter and immune stimulant feed additives.

Key words: Echinacea purpurea, Garlic, Immune response, Broiler chicks.

\section{INTRODUCTION}

For the past several decades, different strategies have been applied to improve poultry productivity and profitability. The most important of them were always directed towards maintaining health, reducing disease outbreak and improving general immunity. Antibiotics growth promoter feed additives have been successfully used at sub therapeutic doses in poultry production to protect health and maximize the efficiency of production, product quality, to control diseases and thegenetic potential of poultry (Landy et al., 2011).

However, their use of Antibiotics as growth promoters has been completely banned throughout Europe and alternative strategies are now needed. In this context, there has been increased interest in the use of biological products, including enzymes, probiotics, prebiotics, synbiotics, organic acids and plant extracts (phytobiotics), as alternatives to antibiotic feed additives in diets for monogastric animals (Bedford, 2000 and Wenk, 2003).

Phytobiotics are plant-derived natural bioactive compounds that can be incorporated into diets in order to enhance the performance and well-being of animals. One of the most important Phytobiotic and popular medical herb is Echinacea purpurea [coneflower] (Barrett, 2003). This herbal medicine has been used from long time ago for a variety of purposes including treatment, growth enhancement and immunostimulation (Percival, 2000).

The positive effects of herbal plants on broilers have been reported by many studies (Tekeli et al., 2006). 
Their anti-biotical potential, hypocholesterolemic effects, growth promoting and availability are the most beneficial part of herbs, which have drawn the scientists' attention. Garlic (Allium sativum), has been approved scientifically for use as antiartherosclerosis, anti-microbial, hypolipidemic, antithrombosis, anti-hypertension, anti-diabetes. Garlic has ajoene, S-allyl cysteine, di allyl (di/tri) sulphide and the most active one allicine (Rahmatnejad et al., 2009). Allicine possibly reduces LDL, triglyceride and cholesterol in serum (Alder and Holub, 1997), hence been used for cardiovascular diseases (Tanamai et al., 2004).

The medicinal herb Echinacea purpurea L. (E.P) is commonly known as an immune stimulating substance which is most widely used as herbal medicines throughout Europe and North America for the prevention or treatment of infectious diseases (Akhtar et al., 2003 and Nasir and Grashorn, 2006). Echinacea and its different derivatives contain a variety of active substances like alkamides, glycoproteins, phenolic compounds, cinnamic acid, essential oil and flavonoids (Barrett, 2003). These substances are effective in treatment of various ailments and proved to be beneficial in improving immunity (Bauer, 1999). However, there have been few reports on the effect of the herb in chickens.

So, the present study aimed to: investigate the supplementation of garlic and Echinacea purpurea in diet on broiler chickens to study their possible effect on performance, immune system and some biochemical blood parameters.

\section{MATERIALS and METHODS}

1-Animals and dietary treatment: 180 , one day old chicks were purchased from Ismailia/Misrpoultry farm, individually weighted, wing banded and randomly divided into 3 maingroups.

Group A: was used as a control group and fed commercial basal diet.

Group B: was fed commercial basal diet plus (5 $\mathrm{mg} / \mathrm{kg}$ feed) Echinacea purpurea (Immunvita-EMA pharm pharmatheutical co.) (Landy et al., 2011).

Group C: was fed commercial basal diet plus ( $1 \mathrm{~g} / \mathrm{kg}$ feed) garlic powder (Kamal and Kassab, 2009)

At day 21, each group was subdivided into two groups as:

group A: group1(G1) and group2(G2), group B: group3(G3) and group4(G4) and group C: group5(G5) and group6(G6).

Groupsof 2,4 and 6 were challenged with $0.5 \mathrm{ml} 24$ hrs broth culture of field strain of E. coli $\mathrm{O} 78$ serotype containing $4 \times 10^{6}$ colony-forming units by intraperitoneal route. The experiment design was illustrated in table (1):

Table 1: Showing design of experiment

\begin{tabular}{|c|c|c|c|c|c|c|}
\hline $\begin{array}{l}\text { Day1 } \\
\mathrm{N}=180\end{array}$ & \multicolumn{2}{|c|}{$\begin{array}{c}\text { Group A } \\
\text { Commercial ration alone }\end{array}$} & \multicolumn{2}{|c|}{$\begin{array}{c}\text { Group B } \\
\text { Commercial ration } \\
5 \mathrm{mg} / \mathrm{kg}+\text { Echinacea } \\
\text { purpurea }\end{array}$} & \multicolumn{2}{|c|}{$\begin{array}{c}\text { Group C } \\
\text { Commercial ration } \\
+ \text { garlic powder } 1 \mathrm{~g} / \mathrm{kg}\end{array}$} \\
\hline Number of chickens & \multicolumn{2}{|c|}{60} & \multicolumn{2}{|c|}{60} & \multicolumn{2}{|c|}{60} \\
\hline Commercial ration & \multicolumn{2}{|c|}{+} & \multicolumn{2}{|c|}{+} & \multicolumn{2}{|c|}{+} \\
\hline Echinacea purpurea $5 \mathrm{mg} / \mathrm{kg}$ & \multicolumn{2}{|c|}{-} & \multicolumn{2}{|c|}{+} & \multicolumn{2}{|c|}{-} \\
\hline garlic powder $1 \mathrm{~g} / \mathrm{kg}$ & \multicolumn{2}{|c|}{-} & \multicolumn{2}{|c|}{-} & \multicolumn{2}{|c|}{+} \\
\hline Day 21 & Group1 & Group2 & Group3 & Group4 & Group5 & Group6 \\
\hline Number of chickens & 30 & 30 & 30 & 30 & 30 & 30 \\
\hline $\begin{array}{l}\text { E. coli inoculation } 4 \times 10^{6} \\
\text { colony-forming }\end{array}$ & - & + & - & + & - & + \\
\hline
\end{tabular}

* Five chickens from each group were subjected for postmortem examination at 2days post infection.

The environmental conditions were equal for every group. Vaccination schedule were carried out against Newcastle disease, Avian influenza, Infectious Bronchitis and Infectious Bursal Disease at recommended age and dose.

\section{2- Live body Performance:}

Body weight was measured in $14^{\text {th }}, 28^{\text {th }}$ and $42^{\text {th }}$ day of experiment. Feed consumption, feed conversion ratio and mortality rate were determined and calculated.

\section{3- Blood samples:}

Blood was collected from wing vein from 10 birds of each group at 7 and 14 days post infection and divided as follow:

EDTA blood: Blood was collected in clean dry bottle containing dipotassium salt of EDTA as 
anticoagulant in concentration of $2 \mathrm{mg} / 1 \mathrm{ml}$ of blood (Jain, 2000) for hematological studies.

Serum: blood was collected in plain clean well-dried centrifuge tube and used for separation of serum to be used in estimation of biochemical parameters.

\section{4- Hematological analysis:}

Total leucocytic (WBCs $10^{3} / \mu \mathrm{l}$ ) and differential leucocytic counts were determined according to routine hematological examination and standard blood smear (Jain, 2000). After that, estimation of Heterophil/Lymphocyte ratio $(\mathrm{H} / \mathrm{L})$ by follow formula: $\mathrm{H} / \mathrm{L}=$ Percent of Hetrophil in blood film /Percent of Lymphocyte in blood film.

\section{5- Serum biochemical analysis:}

The collected sera were assayed for serum biochemistry. The level of alanine aspartate aminotransferase (AST) and alanine aminotransferase (ALT) according to Reitman and Frankel (1957), creatininewas determined according to Henry (1979), uric acid (Caraway, 1963), total serum protein (Henry, 1964), albumin (Doumas, 1971), cholesterol and triglyceride were determined by using ready-made kits from (BioMeriux -France) by Auto analyzer Hitachi 912.

\section{6- Immunologicalstudies:}

a- Weight of lymphoid organs index: Five birds were randomly selected from each subgroup at 42 days of age; slaughtered and lymphoid organs (thymus, spleen and Bursa of Fabricius) were dissected out, weighed and calculated according to the formula of (Halouzka and Jurajda, 1991).

b- Protein electrophoresis: was done using SDSPolyacrylamide gel electrophoresis according to Laemmli (1970).

c- Lymphocyte proliferation assay: Lymphocyte blastogenesis was applied to estimate the effect of Echinacea purpurea and garlic alone or in combination with $E$. coli infection on chicken lymphocyte proliferation. Briefly, the heparinized blood was layered carefully on the surface of lymphocyte separation medium, Ficolhypaque (1:1) in a $5 \mathrm{ml}$ sterile centrifuge tube, then centrifuged for 40 min at 2400 r.p.m. at $4{ }^{\circ} \mathrm{C}$ and the separated Buffy coat was washed with RPMI-1640 medium three times each for $10 \mathrm{~min}$ at 2400, 2000 and 1500 r.p.m., respectively. The sediment washed lymphocytes were re-suspended in one $\mathrm{ml}$ of RPMI-1640 medium containing $10 \%$ fetal calf serum. Then to $100 \mu \mathrm{l}$ of lymphocytes suspension, $100 \mu \mathrm{l}$ of $0.4 \%$ trypan blue were added,. The number of viable lymphocytes were adjusted at a final concentration of $2 \times 10^{6}$ cells/ml according to (Hanks and Wallace, 1985 and Husdan and Hay, 1980) and suspended in RPMT medium containing $10 \%$ fetal calf serum. The phytohaemagglutinin (PHA) was reconstituted in RPMI-medium and the required concentration could be made to $10 \mu \mathrm{g} / \mathrm{ml}$, then filtered through millipore filters ( 0.22 um pore size, Millipore, USA) and store at $-20{ }^{\circ} \mathrm{C}$. Flat bottom sterile tissue culture plates with 96 wells were used as follows: 3 wells containing $100 \mu \mathrm{l}$ of suspended lymphocytes (2X10 cells) in $50 \mu \mathrm{l}$ growth media (RPMI $+10 \%$ fetal calf serum) served as cell control, 3 wells containing 100 $\mu \mathrm{l}$ of suspended lymphocyte $+50 \mu \mathrm{l}$ of PHA mitogen $(15 \mu \mathrm{l} / \mathrm{ml})$ and 3 wells containing $150 \mu \mathrm{l}$ of RPMT1640 medium (medium control), then the plates were incubated at $37^{\circ} \mathrm{C}$ in a humid $\mathrm{CO}_{2}$ atmosphere $(5-$ $10 \% \mathrm{CO}_{2}$ ), then blastogenesis was assayed after 48 $72 \mathrm{hrs}$ using residual glucose in the supernatant of tissue culture medium according to Decock et al. (1980).

7- Statisticalanalysis: After obtaining the data, they were analyzed by variance method (ANOVA) considering $\mathrm{P}<0.05$ using SPSS 18.0 software (2009). The significant differences were taken to Duncan multiple range tests to compare the means.

\section{RESULT}

Experimentally infected non treated chickens of group (G2) showed clinical signs of restlessness, ruffled feathers, loss of appetite, mouth breathing and sneezing $24 \mathrm{hrs}$ post infection. At autopsy of diseased chicks after 48 hour post infection, showed several gross pathological lesionssuch as airsaculitis, severe lung congestion, pericarditis, perihepatitis with kidney and liver congestion and general signs of septicemia with mortality 40\% (12/30). The birds experimentally infected and treated with Echinacea purpurea and garlicpowder displayed less clinical symptoms with the mortality rate $16.66 \%(5 / 30)$. The postmortem lesions were reduced to greater extent as compared to infected untreated birds. The severity of signs was reduced in all infected and treated groups than infected untreated ones.

Table (2) shows the effect of different dietary supplements on performance of broiler chickens. The result showed that all treatments have better final result in comparison with control treatment. Live body weight was significantly different among all experimental groups at different age periods. At 42days of age the heaviest body weight $(\mathrm{P} \leq 0.05)$ was recorded in group 3 and group 5 . Themean body weight of group 2 was significantly decreased $(\mathrm{P} \leq$ 0.05 ) from all other groups. The highest amount of FI and WG was seen in the group 3in comparison with the infected group 2 and infected treated groups $4 \& 6$. The results of live body weight and feed intake are normally a reflection to FCR, therefore, at 1- 42 days of age, Echinacea purpurea and garlicpowder recorded the best FCR (1.19) among all groups. 
Table (3), showed that results of immune organs weight. In respect to Thymus Weight/body Weight (TW/BW), Spleen Weight/Body Weight (SW/BW) and Bursa Weight/Body Weight (BW/BW), the significant differences were found only in SW/BW and BW/BW. The means of spleen weight showed significant differences between the groups. The mean weight ratio of group1, group3, group4, group5 and group6 were highly significant in comparison withgroup2.In respect to bursal weight, the highest ratio was recorded in group3, group4, group5 and group6in comparison with group1 and group2.

Table (4), showed the results of hematological findings, at $1^{\text {st }}$ week (leucocytosis, heterophilia, monocytosis, eosinophilia, significant increase in $\mathrm{H} / \mathrm{L}$ ratio and lymphocyte stimulation index) in infected non treated and infected treated groups while birds which fed on Echinacea purpurea and garlicpowder showed a significant increase in lymphocytic count at 7 day. At 14 days post infection revealed significant increase in lymphocytic count in group2, group3and group5 and significant increase in lymphocyte stimulation index in infected treated groups and non-infected treated groups, while in group 2 significant increase in total leucocytic count all over the observation period.
In table (5) there were significant increases in transaminase ALT and AST enzymes in group2, group4 and group6 as well as hypercholesterolemia from first week were reported. While hypocholesterolemia was observed in Echinacea purpurea and garlicpowdertreated groups. The E. coli infected non treated birds displayed a significant increase in serum uric acid and creatinine levels all over the experiment. The chicks fed on Echinacea purpurea and garlicpowder (group 3 and group 5) induced significant decrease in serum uric acid and creatinine levels at $7^{\text {th }}$ days post infection as compared with infected non treated group (group2) and infected treated groups (group4 and group6). On the other had founded non-significant changes in uric acid and creatinine in all treated groups (group3, group4, group5, and group6) at $14^{\text {th }}$ days post infection in comparison to infected non treated group (group2). Table (6) Protienogram examination revealed hypoproteinemia, hypoalbuminemia, hyperglobulinemia (hyperalphaglobulinemia, hyperbetaglobuliemia and hypergammaglo-buliemia) during the observation period in group 2 at 1 st week post infection as well as non-significant changes in total protein, albumin and globulin at 2 nd week after infection in all groupsbut significant increase in gamma globulin in group 3 and group 5 which treated by Echinacea purpurea and garlicpowder.

Table 2: The effect of dietary supplements of Echinacea purpurea and garlic powder on body weight, weight gain, feed intake, feed conversation ratio and mortality rates of broilers supplemented.

\begin{tabular}{|c|c|c|c|c|c|c|c|}
\hline \multirow{3}{*}{ Groups } & \multicolumn{2}{|c|}{ Group A } & \multicolumn{2}{|c|}{ Group B } & \multicolumn{2}{|c|}{ Group C } & \multirow[t]{2}{*}{ SEM } \\
\hline & G1 & $\mathrm{G} 2$ & G3 & G4 & G5 & G6 & \\
\hline & \multicolumn{7}{|c|}{ Body Weight.(BW) } \\
\hline $14 \mathrm{~d}$ & $290.4 \pm 3.3 b$ & $290.4 \pm 3.3 b$ & $300 \pm 2.4 \mathrm{a}$ & $300 \pm 2.4 \mathrm{a}$ & $301.8 \pm 3.5 \mathrm{a}$ & $301.8 \pm 3.5 \mathrm{a}$ & 5.99 \\
\hline $28 \mathrm{~d}$ & $990.5 \pm 4.5 \mathrm{a}$ & $940.5 \pm 4.2 \mathrm{~d}$ & $994.4 \pm 5.2 \mathrm{a}$ & $960 \pm 7.2 b$ & $990.0 \pm 3.5 \mathrm{a}$ & $965 \pm 6.2 b$ & 17.4 \\
\hline \multirow[t]{2}{*}{42} & $1928.0 \pm 3.5 b$ & $1890 \pm 6.5 \mathrm{~d}$ & $2086 \pm 5.7 \mathrm{a}$ & $1930 \pm 5.9 b c$ & $2074.5 \pm 6.8 \mathrm{a}$ & $1935 \pm 7.1 \mathrm{~b}$ & 34.7 \\
\hline & \multicolumn{7}{|c|}{ Weight Gain (g per bird per day).(DWG) } \\
\hline $14 \mathrm{~d}$ & $17.76 \pm 0.28 \mathrm{a}$ & $17.76 \pm 0.28 \mathrm{a}$ & $19.5 \pm 0.45 \mathrm{a}$ & $19.5 \pm 0.45 \mathrm{a}$ & $20.84 \pm 0.58 \mathrm{a}$ & $20.84 \pm 0.58 \mathrm{a}$ & 2.5 \\
\hline $28 \mathrm{~d}$ & $69.04 \pm 0.38 \mathrm{a}$ & $58.4 \pm 0.5 \mathrm{~b}$ & $69.64 \pm 0.61 \mathrm{a}$ & $60.1 \pm 2.9 \mathrm{~b}$ & $69.88 \pm 0.53 \mathrm{a}$ & $60.3 \pm 7.2 \mathrm{~b}$ & 0.12 \\
\hline \multirow[t]{2}{*}{$42 \mathrm{~d}$} & $91.1 \pm 2.1 \mathrm{~b}$ & $89.1 \pm 2.3 b$ & $99 \pm 3.5 \mathrm{a}$ & $90.2 \pm 3.5 \mathrm{~b}$ & $94.3 \pm 5.3 \mathrm{a}$ & $92.2 \pm 4.3 \mathrm{~b}$ & 1.5 \\
\hline & \multicolumn{7}{|c|}{ feed intake(g/bird/day)(DFI); } \\
\hline $14 \mathrm{~d}$ & $29.2 \pm 3.2 \mathrm{~b}$ & $29.2 \pm 3.2 b$ & $36.8 \pm 3.4 \mathrm{a}$ & $36.8 \pm 3.2 \mathrm{a}$ & $34.9 \pm 4.2 \mathrm{a}$ & $34.9 \pm 4.2 \mathrm{a}$ & 2.2 \\
\hline $28 \mathrm{~d}$ & $148 \pm 4.6 \mathrm{~b}$ & $125 \pm 6.2 \mathrm{~d}$ & $168 \pm 5.1 \mathrm{a}$ & $135 \pm 4 . .7 \mathrm{c}$ & $165 \pm 6.2 \mathrm{a}$ & $136 \pm 6.4 \mathrm{c}$ & 3.0 \\
\hline \multirow[t]{2}{*}{$42 \mathrm{~d}$} & $109 \pm 5.6 \mathrm{a}$ & $95 \pm 3.2 b$ & $117.6 \pm 2.4 \mathrm{a}$ & $110.1 \pm 4.6 \mathrm{a}$ & $115.0 \pm 1.5 \mathrm{a}$ & $109.5 \pm 6.4 \mathrm{a}$ & 4.3 \\
\hline & \multicolumn{7}{|c|}{ Feed Conversation Ratio (g/g)(FCR) } \\
\hline $14 \mathrm{~d}$ & $1.64 \pm 1.5 \mathrm{~b}$ & $1.64 \pm 1.5 \mathrm{~b}$ & $1.88 \pm 2.2 \mathrm{a}$ & $1.88 \pm 2.2 \mathrm{a}$ & $1.67 \pm 2.5 \mathrm{~b}$ & $1.67 \pm 2.5 \mathrm{~b}$ & 0.13 \\
\hline $28 \mathrm{~d}$ & $2.14 \pm 2.4 \mathrm{c}$ & $2.14 \pm 1.8 \mathrm{c}$ & $2.41 \pm 2.4 \mathrm{a}$ & $2.25 \pm 1.5 \mathrm{~b}$ & $2.36 \pm 2.7 \mathrm{a}$ & $2.26 \pm 3.0 \mathrm{~b}$ & 0.10 \\
\hline \multirow[t]{2}{*}{$42 \mathrm{~d}$} & $1.2 \pm 2.1 \mathrm{a}$ & $1.07 \pm 1.7 \mathrm{~b}$ & $1.19 \pm 1.6 \mathrm{a}$ & $1.22 \pm 1.5 \mathrm{a}$ & $1.19 \pm 2.0 \mathrm{a}$ & $1.19 \pm 1.4 \mathrm{a}$ & 0.08 \\
\hline & \multicolumn{7}{|c|}{ Percent of Mortality } \\
\hline $1-42 d$ & $2 \%$ & $40 \%$ & $2 \%$ & $16.66 \%$ & $2 \%$ & $16.66 \%$ & \\
\hline
\end{tabular}

Means $( \pm$ S.E) with different superscript $(\mathrm{a}, \mathrm{b}, \mathrm{c}, \mathrm{d})$ within the same column are significantly different at $\mathrm{p}<0.05$ 
Assiut Vet. Med. J. Vol. 60 No. 140 January 2014

Table 3: Effects of Echinacea purpurea and garlic powder supplemented in diet on immune organs weight index

\begin{tabular}{cccc}
\hline Groups & TW/BW & SW/BW & BW $/$ BW \\
\hline G1 & $0.22 \pm 0.01 \mathrm{a}$ & $0.13 \pm 0.01 \mathrm{~b}$ & $0.19 \pm 0.02 \mathrm{~b}$ \\
\hline $\mathrm{G} 2$ & $0.22 \pm 0.01 \mathrm{a}$ & $0.12 \pm 0.01 \mathrm{c}$ & $0.16 \pm 0.01 \mathrm{c}$ \\
\hline $\mathrm{G} 3$ & $0.22 \pm 0.02 \mathrm{a}$ & $0.14 \pm 0.01 \mathrm{a}$ & $0.22 \pm 0.01 \mathrm{a}$ \\
\hline G4 & $0.22 \pm 0.01 \mathrm{a}$ & $0.14 \pm 0.01 \mathrm{a}$ & $0.22 \pm 0.02 \mathrm{a}$ \\
\hline G5 & $0.22 \pm 0.02 \mathrm{a}$ & $0.14 \pm 0.01 \mathrm{a}$ & $0.21 \pm 0.02 \mathrm{a}$ \\
\hline G 6 & $0.22 \pm 0.02 \mathrm{a}$ & $0.14 \pm 0.01 \mathrm{a}$ & $0.21 \pm 0.01 \mathrm{a}$ \\
\hline L.S.D 0.05 & 0.2 & 0.1 & 0.2 \\
\hline
\end{tabular}

TW/BW; Thymus weight/Body weight, SW/BW; Spleen weight/Body weight, BW/BW;

Bursa weight/Body weight. $\mathrm{a}, \mathrm{b}$ and $\mathrm{c}$ different manuscripts indicated significant differences (PB 0.05)

Table 4: Leucogram \& lymphocyte stimulation index in infected groups by E. coli and treated groups by Echinacea purpurea and garlic powder after one and two weeks. $(\mathrm{n}=10)$

\begin{tabular}{|c|c|c|c|c|c|c|c|c|}
\hline Parameters & Rarameters & $\begin{array}{c}\text { Total } \\
\text { leucocytic } \\
\text { count } \times 10^{3} / \mu 1\end{array}$ & $\begin{array}{l}\text { Lymphocyte } \\
\qquad \times 10^{3} / \mu 1\end{array}$ & $\begin{array}{c}\text { Heterophils } \\
\text { x } 10^{3} / \mu 1\end{array}$ & $\begin{array}{c}\text { Monocytes } \\
\text { x } 10^{3} / \mu 1\end{array}$ & $\begin{array}{c}\text { Eosinophils } \\
\text { x } 10^{3} / \mu 1\end{array}$ & $\mathrm{H} / \mathrm{L}$ ratio & $\begin{array}{c}\text { Lymphocyte } \\
\text { stimulation } \\
\text { index }\end{array}$ \\
\hline \multirow[t]{7}{*}{$\mathbf{1}^{\text {st }}$ week } & G1 & $21.15 \pm 1.6 \mathrm{c}$ & $10.40 \pm 1.13 \mathrm{~b}$ & $9.70 \pm 1.16 \mathrm{~b}$ & $0.45 \pm 0.3 b$ & $0.60 \pm 0.2 b$ & $0.9 \pm 1.2 \mathrm{~b}$ & $1.14 \pm 0.1 \mathrm{~b}$ \\
\hline & $\mathrm{G} 2$ & $32.35 \pm 1.5 \mathrm{a}$ & $11.46 \pm 2.4 \mathrm{a}$ & $18.87 \pm 1.14 \mathrm{a}$ & $1.32 \pm 0.4 \mathrm{a}$ & $0.70 \pm 0.5 \mathrm{a}$ & $1.6 \pm 1.1 \mathrm{a}$ & $1.14 \pm 0.13 b$ \\
\hline & G3 & $28.3 \pm 1.6 \mathrm{~b}$ & $18.18 \pm 1.2 b$ & $9.12 \pm 1.01 \mathrm{~b}$ & $0.35 \pm 0.4 \mathrm{~b}$ & $0.63 \pm 0.3 b$ & $0.5 \pm 1.3 b$ & $1.65 \pm 0.2 \mathrm{a}$ \\
\hline & G4 & $30.36 \pm 1.4 \mathrm{a}$ & $10.5 \pm 2.3 \mathrm{a}$ & $17.8 \pm 3.2 \mathrm{a}$ & $1.4 \pm 0.7 \mathrm{a}$ & $0.76 \pm 0.4 \mathrm{a}$ & $1.7 \pm 0.9 \mathrm{a}$ & $1.18 \pm 0.4 b$ \\
\hline & G5 & $26.12 \pm 1.3 b$ & $17.1 \pm 1.5 b$ & $8.0 \pm 1.2 \mathrm{~b}$ & $0.4 \pm 0.5 b$ & $0.62 \pm 0.2 b$ & $0.5 \pm 0.8 b$ & $1.67 \pm 0.3 \mathrm{a}$ \\
\hline & G6 & $30.68 \pm 1.2 \mathrm{a}$ & $11.25 \pm 13 \mathrm{a}$ & $17.7 \pm 1.9 \mathrm{a}$ & $1.05 \pm 0.5 \mathrm{a}$ & $0.78 \pm 0.3 \mathrm{a}$ & $1.6 \pm 1.0 \mathrm{a}$ & $1.18 \pm 0.23 b$ \\
\hline & L.S.D 0.05 & 3.5 & 2.5 & 3.3 & 0.48 & 0.05 & 0.5 & 0.45 \\
\hline \multirow[t]{7}{*}{$2^{\text {nd }}$ week } & G1 & $12.26 \pm 0.8 b$ & $9.30 \pm 1.2 \mathrm{c}$ & $2.55 \pm 1.3 \mathrm{a}$ & $0.25 \pm 0.3 \mathrm{a}$ & $0.16 \pm 0.2 \mathrm{a}$ & $0.27 \pm 0.1 \mathrm{a}$ & $1.20 \pm 0.1 \mathrm{~b}$ \\
\hline & $\mathrm{G} 2$ & $18.60 \pm 0.5 \mathrm{a}$ & $15.2 \pm 1.3 \mathrm{a}$ & $2.9 \pm 1.01 \mathrm{a}$ & $0.27 \pm 0.4 \mathrm{a}$ & $0.23 \pm 0.5 \mathrm{a}$ & $0.19 \pm 0.05 \mathrm{a}$ & $1.14 \pm 0.13 b$ \\
\hline & G3 & $12.90 \pm 1.3 b$ & $10.1 \pm 1.01 \mathrm{~b}$ & $2.50 \pm 0.9 \mathrm{a}$ & $0.25 \pm 0.5 \mathrm{a}$ & $0.15 \pm 0.3 \mathrm{a}$ & $0.25 \pm 0.3 \mathrm{a}$ & $1.35 \pm 0.2 \mathrm{a}$ \\
\hline & G4 & $12.67 \pm 1.7 \mathrm{~b}$ & $9.20 \pm 3.2 \mathrm{c}$ & $2.60 \pm 2.3 \mathrm{a}$ & $0.35 \pm 0.7 \mathrm{a}$ & $0.22 \pm 0.4 \mathrm{a}$ & $0.28 \pm 0.04 \mathrm{a}$ & $1.34 \pm 0.4 \mathrm{a}$ \\
\hline & G5 & $12.66 \pm 1.1 \mathrm{~b}$ & $10.20 \pm 1.6 \mathrm{~b}$ & $2.40 \pm 1.5 \mathrm{a}$ & $0.30 \pm 0.5 \mathrm{a}$ & $0.16 \pm 0.2 \mathrm{a}$ & $0.24 \pm 0.2 \mathrm{a}$ & $1.37 \pm 0.3 \mathrm{a}$ \\
\hline & G6 & $12.05 \pm 1.2 \mathrm{~b}$ & $9.12 \pm 1.9 \mathrm{c}$ & $2.35 \pm 13 \mathrm{a}$ & $0.35 \pm 0.5 \mathrm{a}$ & $0.23 \pm 0.3 \mathrm{a}$ & $0.26 \pm 0.1 \mathrm{a}$ & $1.34 \pm 0.23 \mathrm{a}$ \\
\hline & L.S.D 0.05 & 1.5 & 1.5 & 1.4 & 0.48 & 0.32 & 0.12 & 0.11 \\
\hline
\end{tabular}

Means ( \pm S.E) with different superscript $(a, b, c, d)$ within the same column are significantly different at $p<0.05$ 
Assiut Vet. Med. J. Vol. 60 No. 140 January 2014

Table 5: Some biochemical parameters in infected groups by E. coli and treated groups by Echinacea purpurea and garlic powder.

\begin{tabular}{|c|c|c|c|c|c|c|c|}
\hline & Parameters & $\begin{array}{c}\text { ALT } \\
\mathrm{u} / 1\end{array}$ & $\begin{array}{c}\text { AST } \\
\mathrm{u} / 1\end{array}$ & $\begin{array}{l}\text { Uric acid } \\
\mathrm{Mg} / \mathrm{l}\end{array}$ & $\begin{array}{l}\text { Creatinine } \\
\mathrm{Mg} / \mathrm{l}\end{array}$ & $\begin{array}{c}\text { Cholesterol } \\
\mathrm{Mg} / 1\end{array}$ & $\begin{array}{c}\text { Triglyceride } \\
\mathrm{Mg} / \mathrm{l}\end{array}$ \\
\hline \multirow[t]{7}{*}{$1^{\text {st }}$ week } & G1 & $5.0 \pm 1.5 b$ & $99.2 \pm 21.3 b$ & $7.9 \pm 0.4 b$ & $0.52 \pm 0.05 b$ & $122.5 \pm 2.3 b$ & $114.5 \pm 13.2 \mathrm{a}$ \\
\hline & G2 & $8.4 \pm 0.8 \mathrm{a}$ & $119.0 \pm 23.3 \mathrm{a}$ & $14.2 \pm 1.3 \mathrm{a}$ & $0.71 \pm 0.05 \mathrm{a}$ & $154 \pm 0.4 \mathrm{a}$ & $115.3 \pm 0.5 \mathrm{a}$ \\
\hline & G3 & $4.07 \pm 1.3 \mathrm{~b}$ & $101.4 \pm 20.1 b$ & $8.1 \pm 1.01 b$ & $0.54 \pm 0.3 b$ & $115 \pm 0.3 b$ & $110.2 \pm 0.3 b$ \\
\hline & G4 & $8.2 \pm 0.7 \mathrm{a}$ & $116.0 \pm 21.3 \mathrm{a}$ & $13.5 \pm 3.2 \mathrm{a}$ & $0.75 \pm 0.04 \mathrm{a}$ & $148 \pm 0.7 \mathrm{a}$ & $110.5 \pm 0.4 b$ \\
\hline & G5 & $4.1 \pm 0.3 b$ & $100 \pm 22.1 b$ & $8.5 \pm 1.6 b$ & $0.52 \pm 0.2 b$ & $112.3 \pm 0.5 b$ & $109.2 \pm 0.2 b$ \\
\hline & G6 & $8.3 \pm 1.2 \mathrm{a}$ & $115.5 \pm 21.1 \mathrm{a}$ & $13.6 \pm 1.9 \mathrm{a}$ & $0.70 \pm 0.1 \mathrm{a}$ & $147 \pm 0.5 \mathrm{a}$ & $109.5 \pm 0.3 b$ \\
\hline & L.S.D $\quad 0.05$ & 2.3 & 9 & 1.2 & 0.12 & 17 & 0.32 \\
\hline \multirow[t]{7}{*}{$2^{\text {nd }}$ week } & G1 & $15.0 \pm 1.9 \mathrm{a}$ & $115.2 \pm 6.3 b$ & $4.4 \pm 0.3 b$ & $0.42 \pm 0.2 b$ & $115.5 \pm 3.2 b$ & $71.8 \pm 2.1 \mathrm{a}$ \\
\hline & G2 & $19.9 \pm 0.7 b$ & $140.0 \pm 8.6 \mathrm{a}$ & $7.1 \pm 0.2 \mathrm{a}$ & $0.55 \pm 0.2 \mathrm{a}$ & $135.8 \pm 5.4 \mathrm{a}$ & $76 \pm 5.0 \mathrm{a}$ \\
\hline & G3 & $15.4 \pm 0.6 \mathrm{a}$ & $117.4 \pm 1.1 \mathrm{~b}$ & $4.1 \pm 1.01 \mathrm{~b}$ & $0.44 \pm 0.1 \mathrm{~b}$ & $96.5 \pm 1.2 \mathrm{c}$ & $61.2 \pm 3.4 b$ \\
\hline & G4 & $16.2 \pm 0.7 \mathrm{a}$ & $126.0 \pm 1.3 b$ & $4.3 \pm 1.2 b$ & $0.45 \pm 0.2 b$ & $96.0 \pm 0.7 \mathrm{c}$ & $63.2 \pm 5.5 b$ \\
\hline & G5 & $14.1 \pm 0.3 \mathrm{a}$ & $116.7 \pm 2.1 b$ & $4.5 \pm 1.6 \mathrm{~b}$ & $0.42 \pm 0.1 \mathrm{~b}$ & $92.2 \pm 0.5 \mathrm{c}$ & $60.2 \pm 6.2 b$ \\
\hline & G6 & $16.3 \pm 1.2 \mathrm{a}$ & $125.1 \pm 1.1 b$ & $4.7 \pm 1.9 b$ & $0.45 \pm 0.2 b$ & $97.0 \pm 0.5 c$ & $65 \pm 3.5 b$ \\
\hline & L.S.D $\quad 0.05$ & 3.5 & 11 & 1.8 & 0.05 & 7 & 6.3 \\
\hline
\end{tabular}

Means $( \pm$ S.E) with different superscript $(a, b, c, d)$ within the same column are significantly different at $\mathrm{p}<0.05$

Table 6: Protienogram on broiler chicks experimentally infected with E. coli and infected treated groups

\begin{tabular}{|c|c|c|c|c|c|c|c|c|}
\hline Parameters & Rarameters & $\begin{array}{l}\text { Total } \\
\text { protein }\end{array}$ & albumin & Globulin & $\begin{array}{c}\text { Beta } \\
\text { globulin } \\
\text { gm/dl }\end{array}$ & $\begin{array}{l}\text { Alpha } \\
\text { globulin } \\
\text { gm/dl }\end{array}$ & $\begin{array}{c}\text { Gamma } \\
\text { globulin } \\
\text { gm/dl }\end{array}$ & $\mathrm{A} / \mathrm{G}$ ratio \\
\hline \multirow[t]{7}{*}{$1^{\text {st }}$ week } & G1 & $2.69 \pm 0.03 a$ & $2.1 \pm 0.04 \mathrm{a}$ & $0.59 \pm 0.02 b$ & $0.18 \pm 0.05 b$ & $0.16 \pm 0.03 b$ & $0.25 \pm 0.03 \mathrm{c}$ & $3.56 \pm 0.01 \mathrm{a}$ \\
\hline & G2 & $2.15 \pm 0.1 \mathrm{~b}$ & $1.38 \pm 0.02 b$ & $0.87 \pm 0.01 \mathrm{a}$ & $0.23 \pm 0.02 \mathrm{a}$ & $0.27 \pm 0.04 \mathrm{a}$ & $0.37 \pm 0.02 b$ & $1.58 \pm 0.03 b$ \\
\hline & G3 & $2.73 \pm 0.03 \mathrm{a}$ & $2.1 \pm 0.01 \mathrm{a}$ & $0.75 \pm 0.01 \mathrm{a}$ & $0.16 \pm 0.01 b$ & $0.15 \pm 0.05 b$ & $0.44 \pm 0.02 \mathrm{a}$ & $3.33 \pm 0.2 \mathrm{a}$ \\
\hline & G4 & $2.34 \pm 0.1 \mathrm{~b}$ & $1.55 \pm 0.05 \mathrm{~b}$ & $0.79 \pm 0.02 \mathrm{a}$ & $0.18 \pm 0.05 b$ & $0.26 \pm 0.03 \mathrm{a}$ & $0.35 \pm 0.01 b$ & $1.96 \pm 0.4 b$ \\
\hline & G5 & $2.96 \pm 0.03 \mathrm{a}$ & $2.2 \pm 0.01 \mathrm{a}$ & $0.76 \pm 0.03 \mathrm{a}$ & $0.17 \pm 0.03 b$ & $0.15 \pm 0.02 b$ & $0.44 \pm 0.01 \mathrm{a}$ & $3.49 \pm 0.3 \mathrm{a}$ \\
\hline & G6 & $2.34 \pm 0.02 b$ & $1.57 \pm 0.05 b$ & $0.77 \pm 0.04 a$ & $0.16 \pm 0.05 b$ & $0.25 \pm 0.05 \mathrm{a}$ & $0.36 \pm 0.02 b$ & $2.04 \pm 0.23 b$ \\
\hline & L.S.D $\quad 0.05$ & 0.3 & 0.5 & 0.12 & 0.3 & 0.8 & 0.5 & 1.2 \\
\hline \multirow[t]{7}{*}{$2^{\text {nd }}$ week } & G1 & $3.91 \pm 0.13 \mathrm{a}$ & $2.13 \pm 0.05 \mathrm{a}$ & $1.78 \pm 0.2 \mathrm{a}$ & $0.64 \pm 0.1 \mathrm{a}$ & $0.47 \pm 0.03 \mathrm{a}$ & $0.67 \pm 0.02 b$ & $1.14 \pm 0.01 \mathrm{a}$ \\
\hline & G2 & $3.80 \pm 0.15 \mathrm{a}$ & $1.94 \pm 0.04 \mathrm{a}$ & $1.86 \pm 0.3 \mathrm{a}$ & $0.67 \pm 0.05 \mathrm{a}$ & $0.49 \pm 0.04 \mathrm{a}$ & $0.70 \pm 0.05 b$ & $0.88 \pm 0.03 \mathrm{a}$ \\
\hline & G3 & $3.94 \pm 0.3 \mathrm{a}$ & $2.1 \pm 0.09 \mathrm{a}$ & $1.84 \pm 0.01 \mathrm{a}$ & $0.55 \pm 0.3 \mathrm{a}$ & $0.41 \pm 0.01 \mathrm{a}$ & $0.88 \pm 0.03 \mathrm{a}$ & $1.04 \pm 0.2 \mathrm{a}$ \\
\hline & G4 & $3.86 \pm 0.33 \mathrm{a}$ & $2.04 \pm 2.3 \mathrm{a}$ & $1.82 \pm 0.2 \mathrm{a}$ & $0.59 \pm 0.04 \mathrm{a}$ & $0.46 \pm 0.04 \mathrm{a}$ & $0.80 \pm 0.04 b$ & $0.96 \pm 0.4 \mathrm{a}$ \\
\hline & G5 & $3.96 \pm 0.3 \mathrm{a}$ & $2.3 \pm 0.04 \mathrm{a}$ & $1.86 \pm 0.6 \mathrm{a}$ & $0.56 \pm 0.2 \mathrm{a}$ & $0.43 \pm 0.05 \mathrm{a}$ & $0.87 \pm 0.02 \mathrm{a}$ & $0.82 \pm 0.3 \mathrm{a}$ \\
\hline & G6 & $3.80 \pm 0.16 \mathrm{a}$ & $2.2 \pm 13 \mathrm{a}$ & $1.80 \pm 0.2 \mathrm{a}$ & $0.58 \pm 0.01 \mathrm{a}$ & $0.42 \pm 0.05 \mathrm{a}$ & $0.80 \pm 0.03 b$ & $0.96 \pm 0.23 \mathrm{a}$ \\
\hline & L.S.D 0.05 & 0.43 & 0.31 & 0.3 & 0.15 & 0.16 & 0.07 & 0.27 \\
\hline
\end{tabular}

Means ( \pm S.E) with different superscript $(a, b, c, d)$ within the same column are significantly different at $p<0.05$ 


\section{DISCUSSION}

Phytogenic feed additives (PFA) (often also called as phytobiotics or botanicals) are plant-derived products, used in animal feeding to improve performance of agricultural livestock throughamelioration of feed properties, promotion of production performance, and improving the quality of animal origin food (Windisch et al., 2008). Echinacea is popular herbal immune-stimulator in North America and Europe, while for theirbeneficial effects on immune system and as natural remedy in many diseases (Nasirand Grashorn, 2009).

Concerning, the effect of supplementation of Echinacea purpurea and garlic in dieton body performance, the results showed a significant improvement $(\mathrm{P} \leq 0.05)$ in live body weight, weight gain and feed intake and feed conversion rate through 5 weeks in Echinacea purpurea and garlic powder feeding groups. This improvement of feed conversion ratio with feeding Echinacea purpurea is in agreement with the findings of Maass et al. (2005) who reported that Echinacea purpurea botanicals (herbs and/or spices), supplementation as feed additive improved feed conversion. Gbenga et al. (2009) found that supplementation of chicken diets with garlic marginally improved weight gain and it was better at high level of supplementation $(5 \mathrm{~g} / \mathrm{kg}$ diet). Meanwhile, there are some controversies on the effect of Echinacea purpurea on feed conversion where, Ma et al. (2009) reported that Echinacea purpurea extract significantly lowered the feed conversionefficiency in broilers.

Birds feed Echinacea purpurea andgarlic in diet and experimentally challenged with $E$. coli developed less response in the form of clinical signs, morbidity and mortalities in comparison to infected non treated group, this may due to inhibitory effect of garlic and Echinacea purpurea in colonization of pathogenic E. coli which agreed with Rahimi et al. (2011) who found that feeding garlic and Echinacea purpurea in diet reduce the intestinal bacterial populations in broiler chickens and the colony forming units of Escherichia coli in digesta of ileo-cecum. In the same context, the fighting ability of chickens against infection may be regard to immunostimulatory effects of garlic and Echinacea purpurea which confirmed in immunological investigations in this study. Antibacterial activity of garlic is due to the inhibition of succinicdehydrogenase via the inactivation of thiol group (Jonkers et al., 1999). Also Allen (2003) and Mass et al. (2005) showed that dried extract of Echinacea purpurea can stimulate the immune system and increase the immune response.

Birds infected and non-treated developed decrease in growth rate, body performance and feed conversion in addition to mortality $40 \%$ and morbidity in the form of airsacculitis, severe lung congestion, pericarditis, perihepatitis with kidney and liver congestion and general signs of septicemia. These results agreed with Russell (2003) and Ask et al. (2006) reported that colibacillosis had adverseeffects on growth and health, growth retardation being the main problem, reducedeating behavior leading to reduce feed intake.

Echinacea purpurea and garlic powder groups showed an elevated ratio of the organs weight/ body weight in comparison to the infected non treated group. This indicates that these two herbs had an immune-stimulating effect against $E$. coli infection when comparing the results of pathological lesions with the organ weights. It may be Suggested that Echinacea purpurea and garlic were antibacterial and immunogenic agent (Nasir and Grashorn, 2006 and Nasir and Grashorn, 2008). Also, Mahmood (2006) reported that the highest ratio of SW/BW and $\mathrm{BW} / \mathrm{BW}$ was recorded in groups containing $1 \%$ garlicpowder in the feed of broiler chickens.

Concerning the hematological results that there were clear at 1st week post infection, leucocytosis, heterophilia, monocytosis and eosinophilia, in infected non treated and infected treated groups, while birds which treated with Echinacea purpurea and garlicpowdershowed a significant increase in total leucocytic count, lymphocytic count and lymphocyte stimulation index. At 14 days post infection revealed significant increase in lymphocytic count in group 2, group 3and group 5 and significant increase in lymphocyte stimulation index in infected treated groups (group $4 \&$ group 6) and non-infected treated groups (group 3\& group 5). The significant increase in lymphocytes might indicate the specific and nonspecific immune stimulant role of Echinacea purpurea and garlic. This is in agreement with Cundell et al. (2003) who found a significant increase of lymphocytes in rats fed with dried Echinacea preparations. Bohmer et al. (2009) reported that ethanolic juice of Echinacea purpurea increased the number of lymphocytes and total leucocytes in hens and pigs. While in group 2 significant increase in total leucocytic count at 14 days after infection. This suggestion is confirmed by those found by Fraser et al. (1991) who stated that leucocytosis, lymphocytosis and monocytosismight be associated with infection.

The results of Hetrophil (H) / Lymphocyte (L) ratio showed that significant differences were evident between groups. Birds in groups (group 2, group 4 and group 6) had the highest ratio at 1st week after infection and the lowest $\mathrm{H} / \mathrm{L}$ ratio was recorded in the Echinacea purpurea group (group 3) and Garlic group (group 5) which suggests that Echinacea purpurea and Garlic are good anti stress factors when 
added. This result agreed to some extent with Kamal and Kassab (2009). Many workers reported that Hetrophil/Lymphocyte (H/L) ratio was anexcellent indicator for stresses in chickens (Gross and Siegal, 1983; Kassab et al., 2000; Mahmood, 2006 and Abuzeed, 2008). The stress may be caused by pathological, nutritional or environmental factors (Al-Murrani et al., 1997 and Amedy, 2008).

The results of biochemical tests indicated that a significant increase in transaminase ALT and AST enzymes were noticed in infected non treated group (group2) all over the experimental period while in infected treated groups (group4 and group6) at $1^{\text {st }}$ week after infection. This increased in serum AST level has been associated with hepatocellular damage in chickens, turkeys and ducks as described by Campbell and Coles (1986) and Thrall et al. (2004). The E. coli infected chicks treated either with Echinacea purpurea or garlic denoted a significant decrease in serum AST and ALT values as compared with infected untreated group (group2) at $2^{\text {nd }}$ weeks after infection. These findings may be attributed to the antioxidant effect of Echinacea purpurea as stated by Zhai et al. (2007) and Nasir and Grashorn (2009) who reported that Echinacea purpurea is an important herb rich in phenolic compounds with antimicrobial and antioxidant activity. The antioxidant activity of phenolic compounds in plants is mainly due to their redox properties and chemical structure which can play an important role in neutralizing free radicals, chelating transitional metals and quenching singlet and triplet oxygen by delocalization or decomposing peroxides. Also, Jackson et al. (2002) who reported the antimicrobial effect of garlic and the antioxidant activity in vivo and in vitro.

From the previously mentioned results which indicates that Echinacea purpurea do not have anynegative effect on liver functions and activity of differentenzymes (Nasir and Grashorn, 2008). Similarly, no significant treatment effect on liverenzymes was observed by Maass et al. (2005) by in-feed application of Echinaceapurpurea preparations to pigs. This shows that Echinacea purpurea supplementation has obviously no harmful effect on health of broilers.

The E. coli infected non treated birds displayed a significant increase in serum uric acid and creatinine levels all over the experiment. These findings are close to those of Mellata et al. (2003) who stated that avian pathogenic $E$. coli belongs to extra intestinal pathogenic group of $E$. coli which causes septicemia in poultry. The chicks fed on Echinacea purpurea and garlic powder (group 3 and group 5) induced significant decrease in serum uric acid and creatinine levels at $7^{\text {th }}$ days post infection as compared with infected non treated group (group2) and infected treated groups (group4 and group6). On the other had founded non-significant changes in uric acid and creatinine in all treated groups (group, group, groupand group 6) at $14^{\text {th }}$ days post infection in comparison to infected non treated group (group2). These results mean improvement in body health of chicks. These improvements may be ought to the kidney protective effect of Echinacea purpurea and garlic owing to its antioxidant effect of its phenolic compounds content.

The serum total cholesterol and Triglycerides concentration were significantlyreduced by dietary with Echinacea purpurea and Garlic compared to the control group. Ologhobo et al. (2008); Metwally (2009) and Mansoub and Nezhady (2011) reported that Garlic has reducing effect on triglyceride and cholestrol level and the best result was obtained in 2 $\%$ of garlic in the commercial basal diet.

The $E$. coli infected non treated and infected treated groups provoked a significant decrease in serum total protein, albumin levels, as compared with control group. These results indicated hepatic damage because liver is responsible for the production of a great proportion of plasma protein (Coles, 1986). The infected and treated with Echinacea purpurea and Garlic elicited non-significant changes in total proteins, albumin and globulin at 2 nd week post treatment as compared with group1; meanwhile these changes are non-significantly increased when compared with infected non medicated group. These results indicating improvements in hepatic functions because of hepatoprotective effect of Echinacea purpurea and Garlic owing to the antioxidant effect of its higher contents of phytophenolic compounds as previously discussed. Also, there was significant increase in serum alpha, beta and gamma globulins of infected non treated group compared to the control group. While there was significant increase in serum alpha and beta globulins in infected treated groups with Echinacea purpurea and garlic compared to control group (group1) at $1^{\text {st }}$ week post infection. Also, showed that significant increase in serum gamma globulins of treated (Echinacea purpurea group and garlic group) non infected group compared to the control group at $1^{\text {st }}$ and $2^{\text {nd }}$ weeks. This result may be due to effect of Echinacea purpurea group and garlic group on immunity. An elevation in the alpha and gamma globulins usually indicates activation of the immune system and is most often due to infection or inflammatory diseases (Butler, 1983). However, Echinacea purpurea fermented juice improve health and immunity of the birds by improving serum globulin contents and stabilizing serum creatine kinase activities (reducing the risk of sudden death syndrome) (Nasir and Grashorn, 2010). Albumin, which is synthesized only in the liver, is the main responsible for maintaining the oncotic blood pressure; it may occurhypoalbuminemia in 
cases of hepatic insufficiency, malnutrition and gastrointestinal disturbances within other conditions (Meyer et al., 1995).

In conclusion Echinacea purpurea and garlic powder feed has an antibacterial effect against Escherichia coli, reduces stress, immunostimulant and improve body performance in broiler chickens.

\section{REFERNCES}

Abuzeed, Y.J. (2008): Evaluation of some properties of immune response in broiler vaccinated with different programs against Newcastle disease. M.Sc. thesis. Collage of Veterinary Medicine, University of Duhok- Kurdistan region- Iraq.

Adler, A.J. and Holub, B.J. (1997): Effect of garlic and fish-oil supplementation on serum lipid and lipoprotein concentrations in hypercholesterolemic men. Am J C/in Nutr 1997; 65: 445-50. P

Akhtar, M.S.; Nasir, Z. and Abid, A.R. (2003): Effect of feeding powdered Nigella sativa L. seeds on poultry egg production and their suitability for human consumption. Veterinarski Arhiv., 73: $181-190$.

Allen, P.C. (2003): Dietary supplementation with Echinacea and development of immunity to challenge infection with coccidian. Parasitol. Res. 91, 74-78.

Al-Murrani, W.G.; Kassab, A. and Al-Sam, H. (1997): Heterophil/lymphocyte ratio as a selection criterion for heat resistance in domestic fowls. British Poultry Science 38: 159-163.

Amedy, V.J.A. (2008): Study on some aspects of immunological properties in heat stressed broiler chickens. M.Sc. thesis. Collage of Veterinary Medicine, University of DuhokKurdistan region- Iraq.

Ask, B.; Van der Waaij, E.H.; Van Eck, J.H.H.; Van Arendonk, J.A.M. and Stegeman, J.A. (2006): Defining susceptibility of broiler chicks to colibacillosis. Avian Pathology 35: 147-153.

Barrett, B. (2003): Medicinal properties of Echinacea: A critical review. Phytomed., 10: 66-86.

Bauer, R. (1999): Chemistry, analysis and immunological investigations of Echinacea phytopharmaceuticals. In Wagner $\mathrm{H}$ (eds) Immunomodulatory agents from plants, Birkhauser Verlag publisher, Berlin, pp. 41-48.

Bedford, M. (2000): Removal of antibiotic growth promoters from poultry diets: implications and strategies to minimize subsequent problems. World's Poultry Science Journal 56, 347-365.

Bohmer, B.M.; Salisch, H.; Paulicks, B.R. and Roth, F.X. (2009): Echinacea purpurea as a potential immunostimulatory feed additive in laying hens and fattening pigs by intermittent application. Livest. Sci. 122: 81-85.

Butler, J.E. (1983): Bovine immunoglobulin's: an augmented review. Vet. Immunol. Immunopathol. 4(1-2): 143-152.

Campbell, T.W. and Coles, E.H. (1986): Avian Clinical Pathology In: Coles, E., Vet. Clin. Pathol., 4th Ed., pp: 279-301.

Caraway, W.T. (1963): Uric acid. Stand Methods Clin. Chem. 1963; 4:239-47.

Chi, M.S.; Koh, E.T. and Steward, T.J. (1982): Effects of garlic on lipid metabolism in rats fed cholesterol or lard. J. Nutr. 112: 241-248.

Coles, E.H. (1986): Veterinary Clinical Pathology, 4th ed. W.B. Saunders Company. United Stated of America, pp: 53-55, 285-286, 435.

Cundell. D.R.; Matrone, M.A.; Ratajczak, P. and Pierce, J.D. (2003): The effect of aerial parts of Echinacea on the circulating white cell levels and selected immune functions of the aging male Sprague-Dawley rat. Int. Immunopharmacol. 3(7): 1041-1048.

Decock, W.; Decree, JVantwaitme, I. and Verhaegfu, H. (1980): Measurement of mitogen stimulation of lymphocytes in a glucose consumption test, J. Immunol. Methods. 33:127-192.

Doumas, B. (1971): Colorimetric determination of serum albumin. Clin. Chem. Acta: pp. 400-403.

Fraser, M.; Bergeron, A.; Mays, A. and Susan, E. Aiello (1991): The Merck Veterinary Manual $7^{\text {th }}$ Ed. Rahway, N.J. U.S.A.

Gbenga E. Onibi*; Oluwatoyin E. Adebisi; Adebowale N. Fajemisin and Ayodeji $V$. Adetunji (2009): Response of broiler chickens in terms of performance and meat quality to garlic (Allium sativum) supplementation. African Journal of Agricultural Research Vol. 4 (5), pp. 511-517, May 2009.

Gross, W. and Siegal, H.S. (1983): Evaluation of the hetropil/lymphocyte ration as measure of stress in chickens. Avian Dis. 27: 972-979.

Halouzka, R. and Jurajda, V. (1991): Morphological expression of immunosupression in poultry. Acta vet. Brno.60: 271-276

Hanks, E. and Wallace, R. (1985): Determination of cell viability. Proc. Exp. Bio. Med. 98: 183-192.

Henry, R.J. (1964): Colorimeteric method for determination of serum total protein, Clinical Chemistry, Harper and Row Publishers, New York, 181.

Henry, R.J. (1979): Colorimeteric methods for determination of serum creatinine. Clinical Chemistry, Principles and techniques, 2nd ed. Harper and Row, pp: 525.

Husdan, A. and Hay, I. (1980): Immunology. Blackwell Scientific Publication, Oxfor. London. 
Jackson, R.; McNeil, B.; Taylor, C.; Holl, G.; Ruff, D. and Gwebu, E.T. (2002): Effect of aged garlic extract on casepase-3 activity, in vitro. Nutritional Neuroscience. 5:287-290.

Jain, N.C. (2000): Schalm's veterinary hematology. 8th Ed. Lea and Febiger, Philadelphia, U.S.A..

Jonkers, D.; Van Den Broek, E. ;Van Dooren, I.; Thijs,C.; Dorant, E.; Hageman, G. and Stobberingh, E. (1999): Antibacterial effect of garlic and omeprazole on Helicobacter pylori. Journal of Antimicrobial Chemotherapy 43: 837-839.

Kamal, A and Kassab, N. (2009): effect of black seeds, garlic powder or enrofloxacin on $E$. coli -induced-airsacculitis in broiler chickens Iraqi Poultry Science Journal.5(1):96-110.

Kassab, A.K.; Elaiwi, H.H. and Al-Murrani, W.K. (2000): Hetropil/ Lymphocyte ration under higher and low tempretuer against Newcastle antigen in chicken. IPAAgre. Res. 10: 184-192.

Laemmli, U.K. (1970): Cleavage of structural proteins during the assembly of the head of bacteriophage T4. Nature 227, 680-685.

Landy, N.; GhalamkariGh, Toghyani, M. and Moattar, F. (2011): The effects of Echinacea purpurea L. (purple coneflower) as an antibiotic growth promoter substitution on performance, carcass characteristicsandhumoral immune response in broiler chickens. ournal of Medicinal Plants Research Vol. 5(11), pp. 2332-2338.

Ma, A.; Shi, W.; Niu, X.; Wang, M. and Zhong, X. (2009): Effects of Echinacea purpurea extract on the immunological response to infectious bursal disease vaccine in broilers. Front. Agr. China. 3:1-5.

Maass, N.; Bauer, J.; Paulicks, B.R.; Böhmer, B.M. and Roth-Maier, D.A. (2005): Efficiency of Echinacea purpurea on performance and immune status in pigs. J. Anim. Phys.Anim. Nutr. 89: 244-252.

Mahmood, S.K. (2006): Immune improvement and production performance of broilers for diseases ND and IBD by employment Nigella, Fenugreek and Garlic. PhD. thesis. University of Baghdad, collage of Veterinary Medicine, Public Health Department.

Mansoub, N.H. and Nezhady, M.A.M. (2011): The effect of using Thyme, Garlic and Nettle on performance, carcass quality and blood parameters.

Mellata, M.; dho-Moulin, M.; Dozois, C.M.; Curtiss, R.; Brown, P.K.; Arne, P.; Bree, A.; Desautles, C. and Fairbrither, J. M.(2003): Role of virulence factors in resistance of avian pathogenic Escherichia coli to serum and in pathogenicity, Infect. Immun., 71 (1): 563-540.
Metwally, M.A.A. (2009): Effects of garlic (Allium sativum) on some antioxidant activities in tilapia nilotica (Oreochromisniloticus). World J. Fish. Marin. Sci. 1(1): 56-64.

Meyer, D.J.; Coles, E.H. and Rich, L.J. (1995): Medicina de LaboratórioVeterinária. São Paulo: Interpretação e Diagnóstico. Roca, 1995: 307.

Nasir, Z. and Grashorn, M.A. (2010): Effects of Echinacea purpurea and Nigella sativa supplementation on broiler performance, carcass and meat quality. Journal of Animal and Feed Sciences, 19: 94-104.

Nasir, Z. and Grashorn, M.A. (2006): Use of Black cumin (Nigella sativa Linn.) as alternative to antibiotics in poultry diets. Tagung Schweineund Geflügelernährung, 9: 210-213.

Nasir, Z. and Grashorn, M.A. (2008): Effects of powdered Nigella sativa seed supplementation in broiler diets on performance and blood metabolism. 64 (2): 451.

Nasir, Z. and Grashorn, M.A. (2009): Echinacea: A potential feed and water additive in poultry and swine production. Arch. Geflügelk. 73(3).

Ologhobo, A.D.; Adebiyi, F.G. and Adebiyi, O.A. (2008): Effect of long term feeding of raw and sun-dried garlic (Allium sativum) on performance and lipid metabolism of broiler chicks. Conference on International Research on Food Security, Natural Resource Management and Rural Development, October 7-9. Tropentag, University of Hohenheim.

Percival, S.S. (2000): Use of Echinacea in medicine. Biochem. Pharmacol. 60(2): 155-158.

Qureshi, A.A.; Abuirmeileh, N.; Din, Z.Z.; Elson, C.E. and Burger, W.C. (1983a): Inhibition of cholesterol and fatty acid biosynthesis in liver enzymes and chicken hepatocytes by polar fractions of garlic. Lipids 18: 343-348.

Qureshi, A.A.; Din, Z.Z.; Abuirmeileh, N.; Burger, W.C.; Ahmad, Y. and Elson, C.E. (1983b): Suppression of avian hepatic lipid metabolism by solvent extracts of garlic: impact on serum lipids. J. Nutr. 113:1746-1755.

Rahimi, S.; Teymouri, Z.; Karimi, M.A.; Omidbaigi, R. and Rokni, H. (2011): Effect of the Three Herbal Extracts on Growth Performance, Immune System, Blood Factors and Intestinal Selected Bacterial Population in Broiler Chickens. J. Agr. Sci. Tech. (2011) Vol. 13: 527-539 527

Rahmatnejad, E.; Roshanfekr, H.; Ashayerizadeh, O.; Mamooee, M. and Ashayerizadeh, A. (2009): Evaluation the Effect of Several NonAntibiotic Additives on Growth Performance of Broiler Chickens. Journal of Animal and Veterinary Advances, 8: 1757-1760.

Reitman, S. and Frankel, S.A. (1957): Colorimetric method for the determination of serum glutamic oxalacetic and glutamic pyruvic 
transaminases. Amer. J. Clin. Pathol. 28: 5663, 1957.

Russell, S.M. (2003): The Effect of Airsacculitis on Bird Weights, Uniformity, Fecal Contamination, Processing Errors and Populations of Campylobacter spp. and E. coli. Poult. Sci. 82: 1326-1331.

SPSS Inc. (2009): PASW STATISTICS 18.0 Command Syntax Reference. SPSS Inc., Chicago

Tanamai, J.; Veeramanomai, S. and Indrakosas, N, (2004): The efficacy of cholesterol-lowering action and side effects of garlic enteric coated tablets in manJ Med Assoc Thai. 87: 1156-1161.

Tekeli, A.; Çelik, L.; Kutlu, H.R. and Gorgulu, M. (2006): Effect of dietary supplemental plant extracts on performance, carcass characteristics, digestive system development, intestinal microflora and some blood parameters of broiler chicks; XII European Poultry Conference, Italy, 2006, 10-14 Sept.
Thrall, M.A.; Baker, D.C.; Campbell, T.W.; DeNicola, D.; fettman, M.J.; Lassen, E.D. and Rebar, A. (2004): Clinical Chemistry of Birds. In: Veterinary Haematology and Clinical Chemistry, Lippincot Williams and Wilkins, U. S. A., P: 479-492.

Wenk, C. (2003): Herbs and botanicals as feed additive in monogastric animals. AsianAustralasian Journal of Animal Science. 16: 282-289.

Windisch, W; Schedle, K.; Plitzner, C. and Kroismayr, A. (2008): Use of phytogenic products as feedadditives for swine and poultry. J. Anim. Sci. 86(E. Suppl.): E140E148.

Zhai, Z.; Yi, L.; WU, L.; Senchina, D.S.; Wurtele, E.S.; Murphy, P.A.; Kohut, M.L. and Cunnik, J.E. (2007): Enhancement of innate and adaptive immune functions by multiple Echinacea species. J. Med. Food 10(3), 423-434.

\section{تأثير حثيثة القنف الارجوانية والثوم على أداء النمو والاستجابة المناعية والقياسات البيوكيميائية والدموية في بداري التسمين}

مايسة محمد غريب ، فاطعة محمد احمد يوسف E

Email: dr-mmg63@yahoo.com

أجريت هذه الدر اسة لدراسة أثر حشيثة القنفذ الارجو انية (بوربوريا يثيناسيا) والثوم على مستوى الاداء فى النمو و الاستجابة المناعية

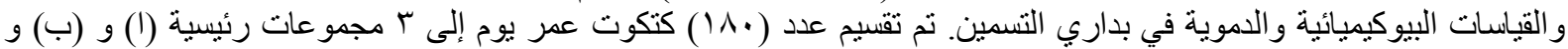

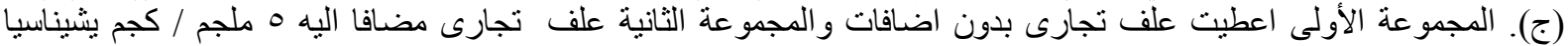

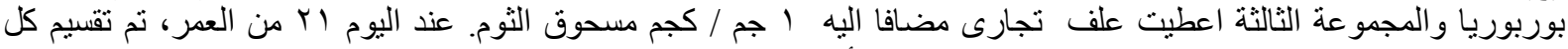

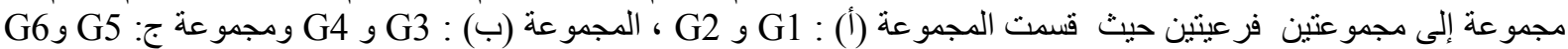

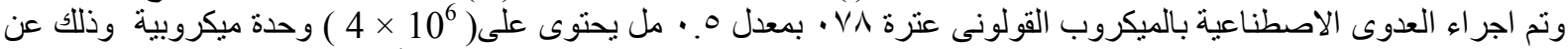

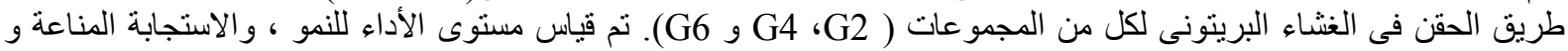

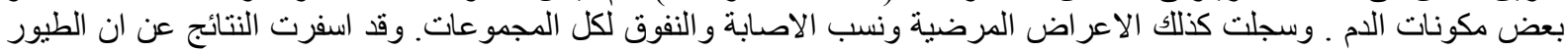

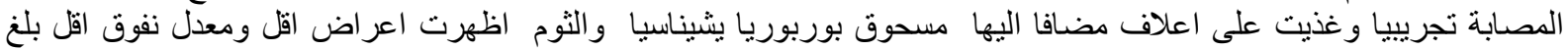

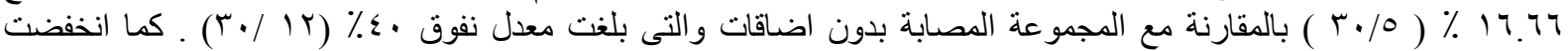

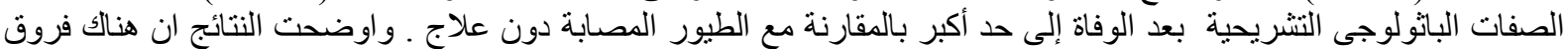

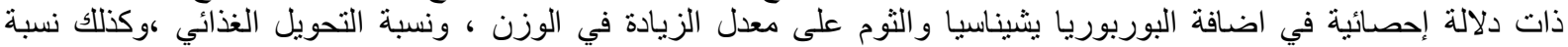

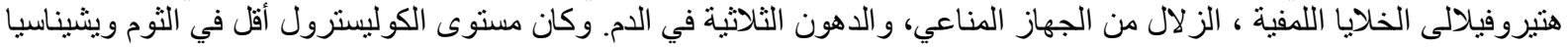

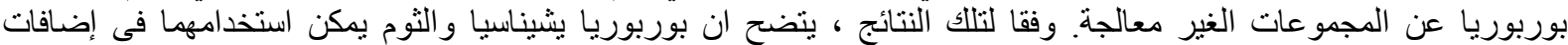
الأعلاف كبديل جيد وامن للمضادات الحيوية ، ومحفز النمو ومنشطا مناعيا. 